\title{
Successes and failures: an overview of lessons learnt in the creation of targeted therapy strategies for breast cancer
}

\author{
S. R. D. Johnston \\ Department of Medicine, Royal Marsden NHS Foundation Trust, Chelsea, London, UK.
}

\begin{abstract}
Therapies that target cancers according to their characteristics (estrogen receptor + ve or -ve, for example) have been a major development in treating cancers successfully and have led to an increase in survival rates. These successes have led to increased research into even more tightly focused therapies. But targeted therapies do not work for everyone, raising issues of (1) identifying patients and (2) developing therapies that reflect our increasing understanding of the complex and differentiated traits of tumors. New targeted therapies such as small molecule signal transduction inhibitors and monoclonal antibodies are being developed to address tumors at all stages of the cell proliferation cycle and it is hoped that they may eventually transform cancer into a fully treatable condition.
\end{abstract}

Keywords: Monoclonal antibodies; Signal transduction inhibitors; Targeted therapy

\section{Introduction}

As our understanding of the pivotal intracellular signals that promote malignant proliferation in breast cancer advances, numerous small molecule signal transduction inhibitors (STIs) and monoclonal antibodies (MoAbs) have been designed to interfere with a number of critical steps along the deregulated intracellular signaling cascade that transmits both proliferative and cell survival signals from the cytosolic membrane on the cell surface down toward the nucleus. Viable targets for which both STI and MoAb therapeutics are in clinical development for breast cancer include growth factor receptors, components of the angiogenesis

Correspondence to: $\mathrm{Dr}$ Stephen R. D. Johnston, MA, FRCP, PhD, Consultant Medical Oncologist, Department of Medicine, Royal Marsden Hospital, Fulham Road, Chelsea, London SW3 6JJ, UK. E-mail: stephen. johnston@rmh.nhs.uk; Tel: +44 207808 2748; Fax: +44 2078082563

Received: $27 / 07 / 07$

Accepted: 13/08/07

BCO/608/2007/FO pathway, intermediate second messengers such as Ras, Raf, MAPK or Src kinases, and cell cycle promoters and transcriptional activators. The various stages of their clinical development are listed in Table 1.

Endocrine therapies have represented the oldest and to date most effective form of targeted therapy in breast cancer. The appropriate selection of patients with estrogen receptor-positive $(E R+v e)$ tumors for treatment with either anti-estrogens (tamoxifen) or estrogen-suppression therapies (ovarian ablation for pre-menopausal women, and aromatase inhibitors for post-menopausal women) has led to significant improvements in survival. The clinical development of endocrine therapy over the last two decades illustrates some of the important lessons that now need to be learnt as the new generation of targeted therapies enter the clinic. Central to these are:

(1) the need to understand the biology around the target, 
Table 1. Targets for small molecule signal transduction inhibitors (STIs) and monoclonal antibodies (MoAb) in breast cancer.

\begin{tabular}{|c|c|c|}
\hline Molecular target & Anti-cancer therapeutic strategy & Stage of clinical development in breast cancer \\
\hline \multicolumn{3}{|l|}{ Growth factor receptors } \\
\hline \multirow{3}{*}{$\begin{array}{l}\text { ErbB family of receptors } \\
\text { EGFR }\end{array}$} & \multirow[t]{3}{*}{ Gefitinib and erlotinib (STI) } & Phase II monotherapy in MBC \\
\hline & & Phase III with chemotherapy in MBC \\
\hline & & Phase III with endo Rx (Tam, Als) in MBC \\
\hline \multirow[t]{3}{*}{ HER2 } & Trastuzumab (MoAb) & Approved in $\mathrm{EBC}$ and $\mathrm{MBC}$ \\
\hline & \multirow[t]{2}{*}{ Lapatinib (STI) } & $\begin{array}{l}\text { Approved (US) with capectibine in trastuzumab } \\
\text { refractory MBC }\end{array}$ \\
\hline & & $\begin{array}{l}\text { Various Phase III trials in EBC \& MBC, either } \\
\text { alone or with chemo/endo } \mathrm{Rx}\end{array}$ \\
\hline All ErbB receptors & Canertinib (STI) & Phase II in MBC \\
\hline \multicolumn{3}{|l|}{ Insulin-like growth factor receptor } \\
\hline \multirow[t]{2}{*}{ IGF1R } & AEW-541 (STI) & Phase I \\
\hline & MK-0646 (MoAB) & Phase $\mathrm{I} / \mathrm{II}$ in $\mathrm{MBC}$ \\
\hline \multicolumn{3}{|c|}{ Vascular endothelial growth factor (VEGFR) } \\
\hline \multirow[t]{3}{*}{ VEGF } & \multirow[t]{3}{*}{ Bevacuzimab (MoAb) } & Approved (EU) with paclitaxel in MBC \\
\hline & & Phase III trials in EBC with chemo \\
\hline & & $\begin{array}{l}\text { Various Phase III trials in MBC either with } \\
\text { chemo/endo Rx/HER2 Rx }\end{array}$ \\
\hline \multicolumn{3}{|l|}{ Multiple growth factor receptors } \\
\hline VEGFR, PDGFR, c-kit and Flt3 & Sunitinib and pazopanib (STI) & $\begin{array}{l}\text { Phase II monotherapy or in combination with } \\
\text { HER2 therapies in MBC }\end{array}$ \\
\hline EGFR, Her2, and VEGFR & AEE-788 & Phase I \\
\hline \multicolumn{3}{|l|}{ Second messengers } \\
\hline \multicolumn{3}{|l|}{ Ras/Raf/Mek/Erk1/2 pathway } \\
\hline Ras & FTls (tipifarnib, lonafarnib) & Phase II/III in MBC \\
\hline Raf & B-RAF inhibitors (sorafinib) & Phase $1 / I I$ in $M B C$ \\
\hline Mek & MEK inhibitors (Cl-1040, PD0325901) & Phase I \\
\hline \multicolumn{3}{|l|}{ PI3K/Akt pathway } \\
\hline \multirow[t]{2}{*}{ mTOR } & Temsirolimus & Phase II/III in MBC \\
\hline & Everolimus & Phase II with Endo Rx in Neoadjuvant EBC \\
\hline Src kinases & AZD0530 & Phase $1 / I I$ in $M B C$ \\
\hline
\end{tabular}

(2) the requirement to identify the target easily in tumors, and to select patients for therapy accordingly, and

(3) the ability to design the most appropriate clinical trial to answer the question asked by the novel targeted therapy.

\section{Clinical development of targeted therapies}

Given the relatively favorable toxicity profile for many of the novel targeted therapies, early dose finding studies should aim to identify the biologically effective dose (BED) rather than the classical maximum tolerated dose (MTD). Pharmacodynamic (PD) endpoints that demonstrate a biological effect on the target and/or its downstream effectors should become an integral part of early clinical development to allow selection of the BED for future studies. This requires the development of reproducible validated assays that can measure the relevant target and its inhibition in tumor samples.
Furthermore, as targeted therapies do not benefit all patients in the same way, appropriate patient selection is needed to minimize the risk of falsenegative trials. Finally, tumor stabilization rather than regression may often be the expected clinical benefit with some of these therapeutics, and thus alternate endpoints to objective tumor response may be appropriate. As discussed below, these considerations are key components for successful clinical trial design with targeted therapies.

\section{Pharmacodynamic endpoints}

Most STIs have favorable toxicity profiles and therefore the MTD may never be reached in conventional phase I studies. Phase I trials should titrate drug dosage to maximal biological effect rather than MTD, which in turn requires the identification of suitable markers of pharmacodynamic or biological activity. One approach is to demonstrate target abolition in paired tumor biopsies pre- and post-treatment. This requires a clear understanding 
of the biological target from pre-clinical studies, and the demonstration that target inhibition actually leads to the desired anti-cancer effect. In the case of HER2 targeted agents such as lapatinib, inhibition of downstream mediators such as pMAPK or pAkt has been shown to correlate with growth inhibition in cell lines, and serial biopsies obtained from patients enrolled in the phase I trial of lapatinib confirmed that baseline elevation in HER2, EGFR, pAkt and MAPK correlated with response [1]. However, paired tumor biopsies can sometimes be technically demanding to obtain in the usual population of patients considered for phase I trials, and efforts have been made to use more easily accessible surrogate tissues such as plasma or skin, although this may not always consistently correlate with intra-tumoral target inhibition. Alternatively, a non-invasive strategy to demonstrate intra-tumoral biological activity is with functional imaging, such as dynamic contrast-enhanced MRI (DCE-MRI) is being used in phase I and II trials of VEGFR targeted therapies to document in vivo changes in vascularization.

\section{Appropriate patient selection}

One of the major challenges in the development of STIs and MoAbs is that not all patients are likely to respond to individualized targeted therapies. The selection of patients most likely to benefit from treatment is therefore pivotal to avoid false-negative studies where a novel therapy may be discarded because it is largely ineffective in an unselected patient population, but potentially valuable in a welldefined subset. For example, had trastuzumab been tested in an unselected population of patients with metastatic breast cancer rather than in the $20 \%$ who have HER 2 + ve tumors, the phase III trials would have been negative and the drug deemed inactive [2]. Likewise, the recent phase III trail of paclitaxel with/without lapatinib was conducted in patients whose tumors were HER2-ve/unknown; overall the trial was negative, yet in the $17 \%$ of patients subsequently found to be HER2 + ve there was a significant treatment effect from the addition of lapatinib [3].

Lack of appropriate target selection is the likely explanation for the disappointing results observed in the various trials of EGFR inhibitors gefitinib and erlotinib in breast cancer. Specific mutations in EGFR can now be used to identify patients who are most likely to respond to treatment with gefitinib, respectively [4]. In addition to activating mutations, over-expression of the target itself or of related upstream or downstream effectors can serve as useful biomarkers. For example, PTEN loss with resulting PI3K up-regulation has been shown to be a powerful predictor of response to mTOR inhibitors in preclinical studies [5]. However, the phase III clinical trial of temsirolimus combined with letrozole vs. letrozole alone in over 1000 endocrine-sensitive metastatic breast cancer patients failed to select for any evidence of pathway activation in the tumors, and was terminated early because it failed to show any benefit for the addition of the STI [6].

Not all STIs have easily identifiable targets, and some compounds such as farnesyltransferase inhibitors (FTIs) or the cell cycle modulators likely inhibit a number of cellular processes, making their specific anti-tumor targets difficult to delineate. Given the lack of proven molecular predictors and our sometimes naiive understanding of the relevant targets, it is critical that any early clinical trials include parallel biological studies to identify the differential molecular characteristics of responding vs. non-responding tumors. The neo-adjuvant setting is especially suited to an in vivo assessment of treatment effect, and can provide valuable insight into biological predictors of response.

\section{Trial design and combination strategies}

Phase II studies traditionally rely on objective response rates to define the anti-tumor effect of a novel treatment. While this may be a suitable endpoint for conventional cytotoxics, lessons learned from completed phase II trials with targeted agents suggest that time to progression (TTP) or stable disease may be more appropriate. However, using stable disease as an endpoint in phase II trial can be challenging when treating naturally slow-growing tumors. The randomized discontinuation design is one approach that can be useful in this setting as it allows investigators to determine if stable disease is attributable to the drug or to the slow-growing nature of the tumor.

Given the complex interaction and redundancy of any aberrant signaling pathway, together with the tumor's ability to develop adaptive mechanisms to evade treatment effect, an argument can be made for combining various targeted agents either with conventional anti-cancer therapies or with complimentary targeted therapies. As we gain greater understanding into the mechanisms of resistance we may rationally use targeted agents in combination or in sequence to delay or abrogate resistance to treatment. For example, the addition of EGFR or HER2 targeted therapies to endocrine therapy in an effort to overcome hormone resistance in breast cancer is the focus of a number of ongoing trials [7]. In vitro studies have demonstrated that in $\mathrm{ER}+\mathrm{ve}$ breast cancer, various growth factor pathways and 
oncogenes involved in the signal transduction cascade become activated and utilized by breast cancer cells to bypass normal endocrine responsiveness [8]. ER remains functional and may engage in cross-talk with a variety of tyrosine kinases such as HER2, Akt and MAPK. Therefore these pathways represent attractive targets for drugs that target these aberrantly expressed signaling pathways. In addition, the therapeutic benefit of vertical combinations of receptor kinase inhibitors with downstream PI3K or mTOR inhibitors is likely to be the subject of future trials.

Given their unique mode of action, targeted therapies may also offer the potential for synergy with conventional chemotherapy at a minimal incremental toxicity cost. The successful combination of the monoclonal antibody trastuzumab with chemotherapy for breast cancer would support such a strategy [2]. More recently, the addition of bevacizumab to chemotherapy has been shown to improve response rates in the treatment of breast cancer, although this did not translate into a survival benefit [9], while the addition of lapatinib to capecitabine chemotherapy improved TTP in breast cancer [10].

It is crucial that studies with new combinations be rationally designed based on robust preclinical data and careful consideration for overlapping toxicities. Many phase II studies of STI combinations have been conducted as single-arm trials, but the obligate experimental design should be the randomized trial design, appropriately powered to detect a meaningful increase in clinical benefit or TTP. In addition, healthy skepticism should be recommended, as strong in vitro synergy may not always translate into synergy in clinical practice.

\section{More cures in early-stage disease?}

Ultimately, targeted therapies may be most appropriate in the minimal residual disease setting after significant tumor debulking has been achieved with conventional chemotherapy or surgery. Additionally, their oral availability (for most STIs) and favorable side-effect profile may make them particularly suited to long-term maintenance. A number of trials are underway to address this issue. Occult quiescent tumor cells have been demonstrated in the serum or bone marrow of breast cancer patients after adjuvant chemotherapy, many in cell cycle arrest as demonstrated by a lack of Ki67 staining [11]. These resting cells respond poorly to standard cytotoxics, but are associated with an increased risk of systemic relapse [12]. While a low proliferation rate makes this residual subpopulation resistant to conventional chemotherapy, these dormant cells may be susceptible to STIs and MoAbs [13], and recently the addition of trastuzumab to adjuvant chemotherapy for women with HER2+ve breast cancer has been shown to significantly improve disease-free survival at 3 years [14].

\section{Conclusion}

As our understanding of the complex biology of neoplasia becomes more sophisticated, small molecule STIs and MoAbs are likely to acquire a critical role in the treatment of most tumor types. Hopefully, lessons learned so far will lead to the rigorous translation of scientific preclinical research into carefully designed human trials that allow us to tailor treatment specifically to the individual patient based on a greater understanding of the molecular mechanisms of sensitivity and resistance. The low-toxicity profile of these novel agents and the judicious use of biological markers of response may transform some advanced cancers into chronic diseases, and ultimately STIs and MoAbs may in fact have an even more promising role in the treatment of early disease with curative intent.

\section{References}

1. Spector NL, Xia W, Burris 3rd H, et al. Study of the biologic effects of lapatinib, a reversible inhibitor of ErbB1 and ErbB2 tyrosine kinases, on tumor growth and survival pathways in patients with advanced malignancies. J Clin Oncol 2005; 23: 2502-2512.

2. Slamon DJ, Leyland-Jones B, Shak S, et al. Use of chemotherapy plus a monoclonal antibody against HER2 for metastatic breast cancer that over-expresses HER2. N Engl J Med 2001; 344: 783-792.

3. DiLeo A, Gomez H, Aziz Z, et al. Lapatinib with paclitaxel versus paclitaxel as first-line treatment for patienst with metastatic breast cancer: a phase III randomized, double-blind study in 580 patients. Proc Am Soc Clin Oncol Abs 2007; Abs 1011.

4. Paez JG, Janne PA, Lee JC, et al. EGFR mutations in lung cancer: correlation with clinical response to gefitinib therapy. Science 2004; 304: 1497-1500.

5. Yu K, Toral-Barza L, Discafani C, et al. mTOR, a novel target in breast cancer: the effect of CCl-779, an mTOR inhibitor, in preclinical models of breast cancer. Endocr Relat Cancer 2001; 8: 249-258.

6. Chow LWC, Shun Y, Jassem J, et al. Phase 3 study of temsirolimus with letrozole or letrozole alone in postmenopausal women with locally advanced or metastatic breast cancer. Breast Cancer Res Treat 2006; 100(Suppl 1); Abs 6091.

7. Johnston SRD. Clinical efforts to combine endocrine agents with targeted therapies against epidermal growth factor receptor/human epidermal growth factor 
receptor 2 and mammalian target of rapamycin in Breast Cancer. Clin Cancer Res 2006; 12: 1061s-1068s.

8. Nicholson RI, McClelland RA, Robertson JF, et al. Involvement of steroid hormone and growth factor cross-talk in endocrine response in breast cancer. Endocr Relat Cancer 1999; 6: 373-387.

9. Miller KD, Chap LI, Holmes FA, et al. Randomized phase III trial of capecitabine compared with bevacizumab plus capecitabine in patients with previously treated metastatic breast cancer. J Clin Oncol 2005; 23: 792-799.

10. Geyer CE, Forster J, Lindquist D, et al. Lapatinib plus capecitabine for HER2-positive advanced breast cancer. N Engl J Med 2006; 355(26): 2733-2743.

11. Braun S, Pantel K, Muller P, et al. Cytokeratin-positive cells in the bone marrow and survival of patients with stage I, II, or III breast cancer. N Engl J Med 2000; 342: 525-533.

12. Braun S, Kentenich C, Janni W, et al. Lack of effect of adjuvant chemotherapy on the elimination of single dormant tumor cells in bone marrow of high-risk breast cancer patients. J Clin Oncol 2000; 18: 80-86.

13. Bozionellou V, Mavroudis D, Perraki M, et al. Trastuzumab administration can effectively target chemotherapyresistant cytokeratin-19 messenger RNA-positive tumor cells in the peripheral blood and bone marrow of patients with breast cancer. Clin Cancer Res 2004; 10: 8185-8194.

14. Romond EH, Perez EA, Bryant J, et al. Trastuzumab plus adjuvant chemotherapy for operable HER2-positive breast cancer. N Engl J Med 2005; 353: 1673-1684. 